\title{
Elevated expression of clonal hematopoiesis of indeterminate potential in patients with coronary endothelial dysfunction is associated with future cardiovascular events
}

Morsaleh Ganji

Mayo Clinic

Terra Lasho

Mayo Clinic

Takumi Toya

National Defense Medical College

Nadia Akhiyat

Mayo Clinic

Changxin Shi

Mayo Clinic

Xianfeng Chen

Mayo Clinic

Esteban Braggio

Mayo Clinic

Ali Ahmad

Mayo Clinic https://orcid.org/0000-0001-9669-8009

Michel Corban

Mayo Clinic

\section{A. Stewart}

Mayo Clinic

Lilach Lerman

Mayo Clinic https://orcid.org/0000-0002-3271-3887

Mrinal Patnaik

Mayo Clinic https://orcid.org/0000-0001-6998-662X

Amir Lerman ( $\square$ lerman.amir@mayo.edu )

Mayo Clinic

Article 
Keywords: Endothelial dysfunction, Clonal hematopoiesis of indeterminate potential, Major adverse cardiovascular events, Inflammatory biomarkers

Posted Date: September 24th, 2020

DOI: https://doi.org/10.21203/rs.3.rs-75002/v1

License: (c) (1) This work is licensed under a Creative Commons Attribution 4.0 International License. Read Full License 


\section{Abstract}

Aims We aimed to test the hypothesis that the presence of clonal hematopoiesis of indeterminate potential (CHIP) in peripheral blood cells is associated with coronary endothelial dysfunction, enhanced inflammatory markers, and major adverse cardiovascular events (MACE).

Methods and results We compared targeted next generation sequencing (35 CHIP related genes) between patients with coronary endothelial dysfunction $(n=123)$ and controls $(n=65)$. Coronary endothelial dysfunction was defined by $\geq 20 \%$ decrease in coronary artery diameter (CAD) or $\leq 50 \%$ increase in coronary blood flow (CBF) in response to acetylcholine injection compared to baseline. Plasma cytokine levels of Interleukin (IL)- 6 and IL-8 were also assessed. Patients were subsequently followed for $12.2 \pm$ 4.3 years. Clonal hematopoiesis relevant gene mutations were found in 1 individual in normal endothelial function group (1.5\%) and 11 cases in endothelial dysfunction group (9.3\%) $(\mathrm{p}=0.04)$. Additionally, CHIP mutations were associated with an increased risk of MACE $(\mathrm{OR}=4.08, P=0.04)$. Mutations in $A S X L 1$, $D N M T 3 A$ and TET2 in the endothelial dysfunction group were also associated with increased levels of IL6 and IL-8 $(P=0.001, P=0.003$; respectively).

Conclusion The current study demonstrates a high frequency of CHIP in patients with coronary endothelial dysfunction as well as an association between mutations in three most common epigenetic regulator genes and increased levels of IL- 6 and IL-8. Therefore it infers a probable relationship between CHIP, endothelial dysfunction and cardiovascular adverse events.

\section{Introduction}

Endothelial dysfunction is the earliest feature of atherosclerosis, which is characterized by decreased bioavailability of nitric oxide (NO) (1). The endothelium is a prime site that is exposed to the atherogenic effects of traditional cardiovascular risk factors (diabetes, hypertension, dyslipidemia, and smoking), biochemical markers and other local anatomic/hemodynamic factors, leading to the development of atherosclerosis (2). Coronary endothelial dysfunction is associated with an increased risk of cardiovascular mortality and myocardial infarction (3). Although traditional cardiometabolic risk factors account for major factors resulting in impairment of endothelial function, many individuals with early coronary atherosclerosis and coronary endothelial dysfunction, especially at younger ages, do not have identifiable comorbidities or risk factors, leading us to propose that unknown elements may play a role in endothelial dysfunction and subsequent cardiovascular diseases (4).

Clonal hematopoiesis of indeterminate potential (CHIP) is defined by the presence of expanded somatic mutations in cancer related genes that arise in hematopoietic stem cells and expand with time, in apparently healthy individuals with no abnormalities in their blood counts (5). It has been demonstrated that approximately $1 \%$ of the population younger than 40 years of age has CHIP, compared to $30 \%$ of the population > 80 years (5). CHIP commonly involved epigenetic regulator genes including DNMT3A (DNA 
methyltransferase 3A), TET2 (Ten-eleven-translocation-2), and ASXL 1 (Additional sex combs-like 1), all of which impact methylation and chromatin dynamics and are associated with inflammation (6). In patients with CHIP, a $40 \%$ increased risk of all-cause mortality surpasses the risk of developing hematological malignancies ( $<1 \% /$ year), and is usually secondary to increased risk of atherosclerotic cardiovascular disease (7). Prior studies on CHIP (7-9) have focused on late stages of atherosclerosis and there is a lack of data to show the impact of CHIP on endothelial dysfunction, which can also be impaired by prevalent inflammation. Yet, patients with mutations in DNMT3A or TET2 are at higher risk of cardiovascular diseases, which is linked to IL-6 receptor activation $(10,11)$. TET2 deficient-cells have also showed increased transcription of IL-6 via alternation in histone deacetylation (12). Based on this, we hypothesize that CHIP is associated with coronary endothelial dysfunction, leading to the development of overt cardiovascular disease. We carried out this study to explore the association between CHIP, inflammation, endothelial dysfunction and adverse cardiovascular events in patients and appropriately matched controls.

\section{Methods}

Mayo Clinic Institutional Review Board approved the current study. Written consent was obtained from all participants. The study was conducted in accordance with the guidelines of the Declaration of Helsinki.

\section{Study population}

In this study, 188 patients who underwent invasive coronary endothelial function testing at Mayo Clinic, Rochester, Minnesota, from 1993 to 2015 were enrolled. Patients presenting with chest pain without history of cardiovascular intervention, myocardial infarction, heart failure, known structural cardiac diseases or evidence of obstructive coronary artery disease on cardiac angiography were selected for physiological assessment of microcirculatory endothelial function by assessment of the change in diameter and blood flow of the coronary artery in response to administration of intracoronary graded infusion of acetylcholine $(13,14)$. Based on the results, patients were categorized into two groups; patients with $(n=123)$ and without $(n=65)$ endothelial dysfunction.

\section{Invasive coronary functional testing}

The study protocol has been described in detail elsewhere $(15,16)$. In brief, patients presenting to the catheterization laboratory with non-obstructive coronary artery (< $40 \%$ stenosis) underwent invasive coronary functional testing using an intracoronary Doppler guidewire. A Doppler guidewire (0.014-inch FloWire, Philips/Volcano Inc) was advanced 2-3 mm distal to the tip of 2.2 F coronary-infusion catheter (Ultrafuse, SciMed Life System) positioned into the mid-portion of the left anterior descending artery (LAD). In all patients, acetylcholine was selectively infused into the LAD at concentrations of $10^{-6}, 10^{-5}$, and $10^{-4} \mathrm{~mol} / \mathrm{L}$ over 3 minutes at each concentration. Doppler measurements and coronary angiography were obtained after each infusion. CAD was measured in segment $5 \mathrm{~mm}$ distal to the tip of the Doppler wire. CBF was calculated from the Doppler-derived time velocity integral and vessel diameter, as 
previously described $(17,18)$. Epicardial endothelial dysfunction was defined as a decrease in CAD of > $20 \%$ in response to acetylcholine as compared to baseline; microvascular endothelial dysfunction was defined as a maximal percentage increase in $\mathrm{CBF}$ in response to acetylcholine $<50 \%$ as compared to baseline $(13,16)$. We defined coronary endothelial dysfunction as either epicardial or microvascular endothelial dysfunction or both.

\section{Clinical and biochemical data}

For all participants prior to undergoing diagnostic tests, demographic and clinical characteristics were obtained through history and physical examination, as previously described $(19,20)$. Data regarding age, sex, body mass index (BMI), smoking status (never/previous/current), hypertension, diabetes and hyperlipidemia were collected. Diabetes was defined as a positive history of diabetes and/or consumption of antidiabetic medications; hypertension was defined as positive history of hypertension and/or consumption of antihypertensive medications; hyperlipidemia was defined as positive history of serum lipid profile out of the normal range and/or usage of lipid lowering medications. Venous blood samples for routine biochemical tests (complete blood count, glucose, creatinine, lipid profile) were obtained after overnight fasting before the procedure. Buffy coat and plasma samples extracted from whole blood were used for collection of DNA and measurement of cytokine expression levels, respectively. Using echocardiography, the percentage of left ventricular ejection fraction was determined and then compared between two study groups. A standardized questionnaire was administered to the patients to record occurrence of MACE and hematologic malignancies in average follow-up of $12.2 \pm 4.3$ years, as was previously described $(19,21)$. Review of medical records was performed blindly by an independent investigator.

\section{Detection of clonal hematopoiesis by targeted capture assays}

DNA was extracted from buffy coat samples following the procedures in Qiagen's Puregene kit. We sequenced the entire coding regions of 35 genes using a customized 150Kb Agilent SureSelect panel. Samples were paired-end sequenced (150 bp reads), using Illumina HiSeq 4000 sequencer with 96 samples per lane of flow cell. The median coverage depth per sample across the 35 genes was $>1000 \mathrm{X}$ per nucleotide, allowing the detection of mutations with variant allelic fraction (VAF) as low as $1 \%$. Raw variants were annotated using GATK Variant Annotator for variant quality, and Biological Reference Repository (BioR) was used for variant annotation (22). Variants with a Mapping Quality $<20$, read depth $<10 \mathrm{X}$, or found in $<1 \%$ of reads were removed. Additionally, sequencing artifacts found in homopolymers were excluded. Finally, variants of significant interest were visually inspected using Integrative Genomics Viewer (IGV) (23).

\section{Primary bioinformatics analysis}

The paired reads of targeted sequencing are mapped to human genome reference $(\mathrm{Hg} 38)$ using BWA (http://bio-bwa.sourceforge.net/bwa.shtml), duplicate reads are marked using picard (https://broadinstitute.github.io/picard/), mutations are called using GATK 
(https://gatk.broadinstitute.org/hc/en-us) by three steps: base recalibration, haplotype caller, and Variant Quality Score Recalibration (VQSR). Finally, mutations are annotated with databases Clinvar (http://www.clinvar.com/), dbSNP (https://www.ncbi.nlm.nih.gov/pmc/articles/PMC29783/) and 1000 genome (https://www.internationalgenome.org/).

\section{Definitions related to Clonal Hematopoiesis Mutations}

Variants were classified as CHIP related if they had a variant allele frequency of $>2 \%$, and exhibited a minor allele frequency of $\leq 0.1 \%$ in the Exome Aggregation Consortium Project (6), a database of known non-somatic variants (ExAC, http://exac.broadinstitute.org/), and were present in the Catalogue of Somatic Mutations in Cancer (COSMIC, https://cancer.sanger.ac.uk/cosmic) database. Additionally, if CHIP mutations were cited in COSMIC as somatic and identified in a hematologic malignancy (known to be pathogenic), we labeled them $\mathrm{CH}-\mathrm{PD}$ (clonal hematopoiesis with a putative driver). Variants below minor allele threshold, and not present in COSMIC, were defined as a variant of uncertain significance (VUS).

\section{Cytokine level analysis}

Plasma samples from 178 patients were tested for the levels of IL- 6 and IL-8 cytokines, by the Cytokine Human Magnetic Kit (cat: LHC0001M, ThermoFisher Scientific) (24). Ten patients were excluded due to missing plasma samples. The bead mix was prepared according to the manufacturer's protocol and samples analyzed in a multiplex array using ProcartaPlex magnetic beads via a Luminex ${ }^{\circledR} 200^{\mathrm{m}}$ instrument (Austin, TX). All plasma samples were run in duplicate. The data were analyzed using the instrument specific software, XPONENT ${ }^{\circledR}$. Statistical comparisons were tabulated using the nonparametric Welch's t test via Prism Software.

\section{Statistical analysis}

In current study, continuous variable are expressed as mean \pm standard deviation (SD). For comparison of quantitative variables between two groups, student t-test was performed for normally distributed variables and Mann-Whitney $U$ test (or Kruskal-Wallis test) for abnormal distributed data. Categorical variable are expressed as frequencies (\%) and analyzed between different groups by the Chi-square test. Univariate and multivariate logistic regression analyses were performed to evaluate the independent association between CHIP and increased risk of MACE as well as association of existence of ASXL1, DNMT3A and TET2 mutations with MACE. For evaluation of association between levels of IL-6 and IL-8 and mutations in ASXL1, DNMT3A and TET2, all study participants were categorized into three groups: individuals with normal endothelial function, individuals with endothelial dysfunction, but without mutations in ASXL1, DNMT3A and TET2 genes and individuals with endothelial dysfunction and mutations in ASXL1, DNMT3A and TET2 genes. These genes were selected from CHIP and VUS groups. Results of regression model were reported as odd ratios (ORs) and $95 \%$ confidence interval ( $95 \% \mathrm{Cls}$ ). A $P$ -value $<0.05$ was assumed for statistical significance. All analyses were perform using SPSS software 
version 25.0 (SPSS Inc., Chicago, IL, USA), GraphPad Prism 6.07 (GraphPad Software, La Jolla, California, USA), and JMP 9 software (SAS Institute, Inc., Cary, NC).

\section{Results}

\section{Baseline characteristics of study participants}

Data from 188 individuals were analyzed. Table 1 summarizes baseline clinical, demographic and biochemical characteristics of the study participants. There was no significant difference in age and sex, cardiovascular risk factors (diabetes, hyperlipidemia, and hypertension), medications use, lipid profiles and other laboratory test results between subjects with impaired versus normal endothelial function. In addition, after an average $12.2 \pm 4.3$ years follow-up, no hematologic malignancies were observed among study participants in either study group.

\section{Association between CHIP and endothelial dysfunction}

In the endothelial dysfunction group, five individuals and in the control group one individual, were excluded because of the lack of sufficient extracted DNA for analysis. In the endothelial dysfunction group, CHIP relevant gene mutations were found in 11 out of $118(9.3 \%)$ remaining individuals (ASXL1, $D N M T 3, C B L$ [casitas B-lineage lymphoma], FLT3-TDK [fms-related tyrosine kinase 3], SETBP1 [SET binding protein 1]) and in 1 out of 64 (1.5\%) individuals (TP53 [tumor protein p53]) in the normal endothelial function group $(P=0.04)$ (Figure 1 and Supplementary table 1); with the prevalence of VUS in CHIP genes being $10.6 \%$ vs $9.2 \%$, respectively $(P=0.77)$. Among these, the most commonly described CHIP mutations including DNMT3A and $A S X L 1(\mathrm{n}=5)$ were found exclusively in patients with endothelial dysfunction.

\section{Associations of CHIP and ASXL1+ DNMT3A+ TET2 mutations with MACE}

Out of 188 individuals, 163 followed-up and completed the questionnaire. In univariate and multivariable logistic regression models, CHIP mutations were associated with an increased risk of subsequent MACE after adjusting for age, sex, hypertension, hyperlipidemia and diabetes $(\mathrm{OR}=3.69, P=0.04$ and $\mathrm{OR}=$ 4.08, $P=0.04$; respectively) (Table 2). Furthermore, in the endothelial dysfunction group, individual mutations in ASXL1, DNMT3A and TET2 were significantly associated with increased risk of MACE by univariate and multivariate regression analyses $(\mathrm{OR}=4.46, P=0.02$ and $\mathrm{OR}=6.17, P=0.01$; respectively) (Table 2).

\section{Association of IL6 and IL-8 level with ASXL1+DNMT3A+TET2 mutations}

Assessment of cytokine levels showed that plasma levels of IL- 6 and IL-8 were both significantly increased in patients with endothelial dysfunction and mutations in ASXL1, DNMT3A and TET2, vs. individuals with normal function of endothelium and individuals in endothelial dysfunction group without mutations in those three genes (IL-6 (median [interquartile range]): $5.53[5.53,11.52]$ vs. $5.53[5.53,5.53]$ 
vs. $5.53[5.53,10.55], p=0.001$; IL-8 (median [interquartile range]): $7.42[7.42,19.19]$ vs. $7.42[7.42,7.42]$ vs. $7.42[7.42,7.42], p=0.003$, respectively) (Figure 2).

\section{Discussion}

The current study demonstrates for the first time a link between the presence of CHIP and early coronary arthrosclerosis, characterized by coronary endothelial dysfunction. Mutations in epigenetic regulator genes ( $A S X L 1, D N M T 3 A$ and TET2) were specifically associated with inflammatory biomarkers (increased plasma levels of IL- 6 and IL-8). Moreover, the existence of this triad mutant CHIP was independently associated with MACE in follow-up observations. The current study therefore supports a potential role for $\mathrm{CHIP}$ in the mechanism of coronary artery disease starting at an early stage of atherosclerosis formation.

Vascular endothelial cells, which regulate vascular tone to maintain blood supply to the tissues and protect vessels from mechanical and chemical stress, constantly undergo injury and repair. Impaired repair damages hemostasis and initiates a variety of changes such as increased vascular permeability and cytokine release that promote atherogenesis. Recent evidence suggests that circulating endothelial progenitor cells arising from the bone marrow play an important role in the repair process of the injured endothelial layer, and impaired function of these cells contributes to endothelial dysfunction $(25,26)$. In addition, circulating monocytes adhere to endothelium and infiltrate into the vessel wall, where they differentiate into various phenotypes of macrophages which play a crucial role in all stages of plaque formation and development of cardiovascular events $(27,28)$.

With age, hematopoietic stem cells acquire mutations which can form clonal populations of mutant peripheral blood cells. Most carriers of these clones have normal blood counts, no evidence for an underlying hematological neoplasm, and in fact will never develop a hematologic neoplasm (CHIP); however they do have a significant increase in mortality (27) and cardiovascular diseases (7). Further, CHIP is associated with degenerative calcified aortic valve stenosis (29) and with worse clinical outcomes in heart failure patients with ischemic cardiomyopathy (30).

The underlying mechanisms of the link between CHIP and cardiovascular diseases are being sought in cardiovascular research. It has been suggested that CHIP associated mutations can alter transcription of genes related to inflammatory pathways in peripheral blood cells such as monocytes, potentially augmenting inflammatory responses during the atherogenesis (10). Murine models of TET2 and DNMT3A loss-of-function mutations showed advanced cardiovascular diseases, potentially through accelerating inflammation (31). A recent observational study supported this hypothesis by showing that in DNMT3A and TET2 associated CHIP, the increased risk of cardiovascular events was mitigated in patients with IL-6 receptor mutations (11). It has been demonstrated that loss of TET2 function in macrophages mediates upregulation of several inflammatory markers such as IL-6 and IL-8 via alterations in DNA methylation, hydroxymethylation and histone deacetylation $(7,32,33)$. Increased levels of both IL- 6 and IL-8 in the microenvironment of vessels can initiate atherosclerosis, as phenotype transformation of cells results in proliferation of vascular smooth muscle cells, endothelial dysfunction 
and activation of pro-inflammatory macrophages (34). Specifically, IL-8 overexpression accompanies increased endothelial permeability and early stages of plaque formation (35). Importantly, our analysis revealed a relationship between mutations in ASXL1, DNMT3A and TET2 genes in patients with endothelial dysfunction and increased plasma levels of IL- 6 and IL-8. This suggests possible mechanistic role of these mutations in inflammatory pathways and initiation or progression of endothelial dysfunction.

In the current study, we observed an increased frequency of CHIP mutations in patients with early coronary atherosclerosis and endothelial dysfunction, especially involving ASXL1. Previous studies have demonstrated a potential role for CHIP in the late stage of coronary artery disease $(7,9,30)$. The current study extends these previous observations by evaluating CHIP associated mutations in patients with the early stage of coronary artery disease prior to the development of any obstructive plaque characterized by coronary endothelial dysfunction. We observed an association of CHIP mutations in ASXL1, DNMT3A, $C B L, F L T 3-T D K$ and SETBP1 with coronary endothelial dysfunction. Moreover, the relationship of these mutations associated CHIP and existence of ASXL1, DNMT3A and TET2 mutations, individually in endothelial dysfunction group with occurrence of myocardial infarction, stroke and death appears to be causal linkage. Consistent with our findings, association of cardiovascular disease with CHIP mutations in DNMT3A and ASXL 1 has been reported in different studies $(7,36)$.

\section{DNMT3A and ASXL1 mutations associated CHIP observed in patients with endothelial dysfunction}

DNMT3A encodes DNA methyltransferase, which adds methyl groups to DNA. This is necessary for maturation of hematopoietic stem cells and their differentiation into different peripheral blood cells. Bone marrow-derived macrophages with loss of function in DNMT3A revealed increased expression of CXC chemokines and synthesis of pro-inflammatory cytokines, IL-6 and IL-1b (11). In addition, Mast cell activation and increased synthesis of interferon $-\gamma$ via T cells were linked to DNMT3A deficiency. Interestingly, mutations in $D N M T 3 A$ gene may also cause T cell polarization into pro-inflammatory and pro-atherogenic type $(8,37)$.

Another common CHIP mutation in epigenetic regulator genes is $A S X L 1$ gene, encoding additional sex combs like 1 protein which influences histone modifications and gene expression (38). Mutation in ASXL 1 gene can result in inhibition of polycomb repressive complex 2 (PRC2)-mediated histone $\mathrm{H} 3$ lysine 27 (H3K27) tri-methylation. This effect causes dysregulation of hematopoietic cells. Loss of function of ASXL 1 leading to CHIP has been investigated for its role in the development of malignant myeloid diseases such as myeloproliferative neoplasms, myelodysplastic syndromes, and acute myeloid leukemia. However, to date, the role of CHIP mutation in ASXL 1 on the development of cardiovascular diseases is still unknown (39).

Based on our results, we postulate that the CHIP mutations may occur especially in epigenetic modulator genes such as $A S X L 1$ of progenitor cells of monocyte or endothelial cells in bone marrow and clonally expand to the circulation (9). After migration of these cells to the arterial wall, expression of genes 
involved in pro-inflammatory mechanisms are elevated due to alternations in methylation of DNA and other epigenetic factors. This process may drive endothelial dysfunction and atherosclerosis formation.

The current study had several limitations. The plasma and buffy coat samples available to us for this current study were obtained at baseline at the time of presentation. Thus, we are unable to evaluate alternations in frequency of mutations overtime. Future studies with large sample size are required to explore underlying mechanisms linking the impact of CHIP on endothelial dysfunction to IL-6 and IL-8. Despite these limitations, the present study is strengthened by investigation of somatic mutations in hematopoietic cells in carefully phenotyped patients with endothelial dysfunction before developing obstructive coronary diseases for the first time in cardiovascular research. Importantly, we assessed the independent association between these mutations and adverse cardiovascular events.

In conclusion, the current study revealed, for the first time, increased somatic mutations related to CHIP in individuals with coronary endothelial dysfunction but without obstructive coronary disease, especially in ASXL 1 gene, postulating a novel mechanism of developing coronary endothelial dysfunction. These mutations may contribute to the progression of cardiovascular diseases, leading to death, myocardial infarction and stroke. Furthermore, enhanced expression levels of IL-6 and IL-8 seems to be related to mutations in DNMT3A, ASXL1 and TET2, more than other gene mutations relevant CHIP. The current study supports a role for CHIP as a mechanism and potential therapeutic target for patients with early coronary atherosclerosis, and advances our understanding of the pathogenesis of the disease.

\section{Declarations}

\section{Acknowledgments}

The authors acknowledge the Center for Individualized Medicine at Mayo Clinic and the Henry Predolin Leukemia Foundation for providing services. We thank Laura A. Bruins in the Department of Hematology at Mayo Clinic, for her help with DNA extraction.

\section{Disclosures}

Dr Patnaik has served on the advisory boards for stem line pharmaceuticals and Kura Oncology.

\section{Competing Interests}

The authors have no competing interests to disclose as defined by Nature Research policy.

\section{Sources of Funding}

This work was supported by grants from National Institute of Health [DK120292, DK122734] and Mayo Foundation.

\section{References}


1. Lerman A, Zeiher AM. Endothelial function: cardiac events. Circulation. 2005;111(3):363-8.

2. Gutierrez E, Flammer AJ, Lerman LO, Elizaga J, Lerman A, Fernandez-Aviles F. Endothelial dysfunction over the course of coronary artery disease. European heart journal. 2013;34(41):3175-81.

3. Jespersen L, Hvelplund A, Abildstrom SZ, Pedersen F, Galatius S, Madsen JK, et al. Stable angina pectoris with no obstructive coronary artery disease is associated with increased risks of major adverse cardiovascular events. European heart journal. 2012;33(6):734-44.

4. Bonetti PO, Lerman LO, Lerman A. Endothelial dysfunction: a marker of atherosclerotic risk. Arteriosclerosis, thrombosis, and vascular biology. 2003;23(2):168-75.

5. Steensma DP, Bejar R, Jaiswal S, Lindsley RC, Sekeres MA, Hasserjian RP, et al. Clonal hematopoiesis of indeterminate potential and its distinction from myelodysplastic syndromes. Blood. 2015;126(1):9-16.

6. Jaiswal S, Ebert BL. Clonal hematopoiesis in human aging and disease. Science (New York, NY). 2019;366(6465).

7. Jaiswal S, Natarajan P, Silver AJ, Gibson CJ, Bick AG, Shvartz E, et al. Clonal Hematopoiesis and Risk of Atherosclerotic Cardiovascular Disease. The New England journal of medicine. 2017;377(2):11121.

8. Yura Y, Sano S, Walsh K. Clonal Hematopoiesis: A New Step Linking Inflammation to Heart Failure. JACC Basic to translational science. 2020;5(2):196-207.

9. Libby P, Sidlow R, Lin AE, Gupta D, Jones LW, Moslehi J, et al. Clonal Hematopoiesis: Crossroads of Aging, Cardiovascular Disease, and Cancer: JACC Review Topic of the Week. Journal of the American College of Cardiology. 2019;74(4):567-77.

10. Cook EK, Luo M, Rauh MJ. Clonal hematopoiesis and inflammation: Partners in leukemogenesis and comorbidity. Experimental hematology. 2020;83:85-94.

11. Bick AG, Pirruccello JP, Griffin GK, Gupta N, Gabriel S, Saleheen D, et al. Genetic Interleukin 6 Signaling Deficiency Attenuates Cardiovascular Risk in Clonal Hematopoiesis. Circulation. 2020;141(2):124-31.

12. Zhang Q, Zhao K, Shen Q, Han Y, Gu Y, Li X, et al. Tet2 is required to resolve inflammation by recruiting Hdac2 to specifically repress IL-6. Nature. 2015;525(7569):389-93.

13. Hasdai D, Holmes DR, Jr., Higano ST, Burnett JC, Jr., Lerman A. Prevalence of coronary blood flow reserve abnormalities among patients with nonobstructive coronary artery disease and chest pain. Mayo Clinic proceedings. 1998;73(12):1133-40.

14. Herrmann J, Lerman A. The endothelium: dysfunction and beyond. Journal of Nuclear Cardiology. 2001;8(2):197-206.

15. Cannon RO, 3rd. Does coronary endothelial dysfunction cause myocardial ischemia in the absence of obstructive coronary artery disease? Circulation. 1997;96(10):3251-4.

16. Widmer RJ, Flammer AJ, Herrmann J, Rodriguez-Porcel M, Wan J, Cohen P, et al. Circulating humanin levels are associated with preserved coronary endothelial function. American journal of physiology 
Heart and circulatory physiology. 2013;304(3):H393-7.

17. Ofili EO, Labovitz AJ, Kern MJ. Coronary flow velocity dynamics in normal and diseased arteries. The American journal of cardiology. 1993;71(14):3d-9d.

18. Bonetti PO, Pumper GM, Higano ST, Holmes DR, Jr., Kuvin JT, Lerman A. Noninvasive identification of patients with early coronary atherosclerosis by assessment of digital reactive hyperemia. Journal of the American College of Cardiology. 2004;44(11):2137-41.

19. Targonski PV, Bonetti PO, Pumper GM, Higano ST, Holmes Jr DR, Lerman A. Coronary endothelial dysfunction is associated with an increased risk of cerebrovascular events. Circulation. 2003;107(22):2805-9.

20. Sara JD, Widmer RJ, Matsuzawa Y, Lennon RJ, Lerman LO, Lerman A. Prevalence of Coronary Microvascular Dysfunction Among Patients With Chest Pain and Nonobstructive Coronary Artery Disease. JACC Cardiovascular interventions. 2015;8(11):1445-53.

21. Suwaidi JA, Hamasaki S, Higano ST, Nishimura RA, Holmes Jr DR, Lerman A. Long-term follow-up of patients with mild coronary artery disease and endothelial dysfunction. Circulation. 2000;101(9):94854.

22. Kocher JP, Quest DJ, Duffy P, Meiners MA, Moore RM, Rider D, et al. The Biological Reference Repository (BioR): a rapid and flexible system for genomics annotation. Bioinformatics (Oxford, England). 2014;30(13):1920-2.

23. Robinson JT, Thorvaldsdottir H, Winckler W, Guttman M, Lander ES, Getz G, et al. Integrative genomics viewer. Nat Biotechnol. 2011;29(1):24-6.

24. Lynch HE, Sanchez AM, D'Souza MP, Rountree W, Denny TN, Kalos M, et al. Development and implementation of a proficiency testing program for Luminex bead-based cytokine assays. Journal of immunological methods. 2014;409:62-71.

25. GossI M, Modder UI, Gulati R, Rihal CS, Prasad A, Loeffler D, et al. Coronary endothelial dysfunction in humans is associated with coronary retention of osteogenic endothelial progenitor cells. European heart journal. 2010;31(23):2909-14.

26. Boilson BA, Kiernan TJ, Harbuzariu A, Nelson RE, Lerman A, Simari RD. Circulating CD34+ cell subsets in patients with coronary endothelial dysfunction. Nature clinical practice Cardiovascular medicine. 2008;5(8):489-96.

27. Li F, Wu X, Zhou Q, Zhu DW. Clonal hematopoiesis of indeterminate potential (CHIP): A potential contributor to atherlosclerotic cardio/cerebro-vascular diseases? Genes \& diseases. 2018;5(2):75-6.

28. Dansky HM, Barlow CB, Lominska C, Sikes JL, Kao C, Weinsaft J, et al. Adhesion of monocytes to arterial endothelium and initiation of atherosclerosis are critically dependent on vascular cell adhesion molecule-1 gene dosage. Arteriosclerosis, thrombosis, and vascular biology. 2001;21(10):1662-7.

29. Mas-Peiro S, Hoffmann J, Fichtlscherer S, Dorsheimer L, Rieger MA, Dimmeler S, et al. Clonal haematopoiesis in patients with degenerative aortic valve stenosis undergoing transcatheter aortic valve implantation. European heart journal. 2020;41(8):933-9. 
30. Libby P, Jaiswal S, Lin AE, Ebert BL. CHIPping Away at the Pathogenesis of Heart Failure. JAMA cardiology. 2019;4(1):5-6.

31. Sano S, Oshima K, Wang Y, Katanasaka Y, Sano M, Walsh K. CRISPR-Mediated Gene Editing to Assess the Roles of Tet2 and Dnmt3a in Clonal Hematopoiesis and Cardiovascular Disease. Circulation research. 2018;123(3):335-41.

32. Cook EK, Izukawa T, Young S, Rosen G, Jamali M, Zhang L, et al. Comorbid and inflammatory characteristics of genetic subtypes of clonal hematopoiesis. Blood advances. 2019;3(16):2482-6.

33. Zhang Q, Zhao K, Shen Q, Han Y, Gu Y, Li X, et al. Tet2 is required to resolve inflammation by recruiting Hdac2 to specifically repress IL-6. Nature. 2015;525(7569):389-93.

34. Liu Y, Peng W, Qu K, Lin X, Zeng Z, Chen J, et al. TET2: A Novel Epigenetic Regulator and Potential Intervention Target for Atherosclerosis. DNA and cell biology. 2018;37(6):517-23.

35. Yu H, Huang X, Ma Y, Gao M, Wang O, Gao T, et al. Interleukin-8 regulates endothelial permeability by down-regulation of tight junction but not dependent on integrins induced focal adhesions. International journal of biological sciences. 2013;9(9):966-79.

36. Haybar H, Shahrabi S, Ghanavat M, Khodadi E. Clonal hematopoiesis: Genes and underlying mechanisms in cardiovascular disease development. Journal of cellular physiology. 2019;234(6):8396-401.

37. Libby P, Ebert BL. CHIP (Clonal Hematopoiesis of Indeterminate Potential): Potent and Newly Recognized Contributor to Cardiovascular Risk. Circulation. 2018;138(7):666-8.

38. Micol JB, Abdel-Wahab O. The Role of Additional Sex Combs-Like Proteins in Cancer. Cold Spring Harbor perspectives in medicine. 2016;6(10).

39. Nagase R, Inoue D, Pastore A, Fujino T, Hou HA, Yamasaki N, et al. Expression of mutant Asxl1 perturbs hematopoiesis and promotes susceptibility to leukemic transformation. The Journal of experimental medicine. 2018;215(6):1729-47.

\section{Tables}

Table 1. Baseline biochemical, demographic and clinical characteristics of study participants between endothelial dysfunction and normal function groups. 


\begin{tabular}{|c|c|c|c|}
\hline Parameters & $\begin{array}{l}\text { Patients with normal } \\
\text { endothelial function } \\
(n=65)\end{array}$ & $\begin{array}{l}\text { Patents with endothelial } \\
\text { dysfunction } \\
(n=123)\end{array}$ & $\begin{array}{l}P \\
\text { value }\end{array}$ \\
\hline Age,(years) & $51.93 \pm 10.11$ & $53.78 \pm 10.22$ & 0.238 \\
\hline Sex, n, M/F & $14 / 51$ & $40 / 83$ & 0.113 \\
\hline $\mathrm{BMI},\left(\mathrm{kg} / \mathrm{m}^{2}\right)$ & $50.76 \pm 6.25$ & $29.40 \pm 5.97$ & 0.175 \\
\hline $\mathrm{Hb}(\mathrm{g} / \mathrm{dL})$ & $13.3 \pm 1.2$ & $13.6 \pm 1.24$ & 0.142 \\
\hline $\mathrm{MCV}(\mathrm{fL})$ & $89.1 \pm 4.6$ & $88.41 \pm 8.38$ & 0.519 \\
\hline WBC $\left(x 10^{9} / \mathrm{L}\right)$ & $6.44 \pm 2.21$ & $6.40 \pm 1.77$ & 0.893 \\
\hline PLT (x109/L) & $253.60 \pm 80.16$ & $247.99 \pm 59.81$ & 0.597 \\
\hline Diabetes, n (\%) & $5(7 \%)$ & $10(8 \%)$ & 0.951 \\
\hline Hypertension, n (\%) & $29(46 \%)$ & $50(40 \%)$ & 0.571 \\
\hline Hyperlipidemia, n (\%) & $32(50 \%)$ & $75(61 \%)$ & 0.144 \\
\hline $\begin{array}{l}\text { Smoking, n } \\
\text { (never/previous/current) }\end{array}$ & $34 / 24 / 4$ & $73 / 39 / 9$ & 0.681 \\
\hline Total Cholesterol, mg/dL & $182.65 \pm 39.02$ & $185.73 \pm 39.09$ & 0.627 \\
\hline HDL-Cholesterol, mg/dL & $58.35 \pm 17.27$ & $55.26 \pm 17.60$ & 0.271 \\
\hline LDL- Cholesterol, mg/dL & $100.06 \pm 30.38$ & $105.39 \pm 30.38$ & 0.325 \\
\hline Triglycerides, mg/dL & $121.23 \pm 72.72$ & $131.39 \pm 118.28$ & 0.547 \\
\hline Creatinine, $\mathrm{mg} / \mathrm{dL}$ & $1.24 \pm 2.40$ & $0.97 \pm 0.19$ & 0.387 \\
\hline Glucose, mg/dL & $100.85 \pm 18.58$ & $100.59 \pm 22.95$ & 0.937 \\
\hline Aspirin, $\mathrm{n}(\%)$ & $34(54 \%)$ & $75(62 \%)$ & 0.294 \\
\hline$\beta$ - Blocker, n (\%) & $19(30 \%)$ & $50(41 \%)$ & 0.149 \\
\hline $\begin{array}{l}\text { Lipid - lowering } \\
\text { medications, n (\%) }\end{array}$ & $26(41 \%)$ & $60(49 \%)$ & 0.307 \\
\hline Nitrates, n (\%) & $23(36 \%)$ & $44(36 \%)$ & 0.953 \\
\hline $\begin{array}{l}\text { Echocardiography ejection } \\
\text { fraction }(\%)\end{array}$ & $64.17 \pm 8.25$ & $61.95 \pm 7.80$ & 0.28 \\
\hline
\end{tabular}

For continuous variables, data was expressed as mean $\pm \mathrm{SD}$. BMI, body mass index; HDL-C, high-density lipoproteins cholesterol; LDL-C, low-density lipoproteins cholesterol; WBC, white blood cells; HGB, hemoglobin; MCV, mean corpuscular volume, PLT; platelet 
Table 2. Association of CHIP and ASXL1+DNMT3A+TET2 mutations with MACE in univariate and multivariate logistic regression analysis

\begin{tabular}{|llll|}
\hline Univariate & \multicolumn{3}{c|}{ death+Ml+stroke } \\
\hline & OR & $95 \% \mathrm{Cl}$ & $P$ value \\
\hline CHIP & 3.69 & {$[1.05,12.95]$} & 0.04 \\
\hline ASXL 1+DNMT3A+TET2 & 4.46 & {$[1.21,16.47]$} & 0.02 \\
\hline & & & \\
\hline Multivariate & & death+Ml+stroke & \\
\hline & adjusted OR & $95 \% \mathrm{Cl}$ & $P$ value \\
\hline CHIP & 4.08 & {$[1.05,15.85]$} & 0.04 \\
\hline ASXL 1+DNMT3A+ TET2 & 6.17 & {$[1.43,26.56]$} & 0.01 \\
\hline
\end{tabular}

Multivariate logistic regression model is adjusted for age, gender, hypertension, hyperlipidemia and diabetes. CHIP, clonal hematopoiesis of indeterminate potential; MI, myocardial infarction.

Odds ratios (ORs) and 95\% confidence intervals (Cls) were reported for each model.

\section{Figures}

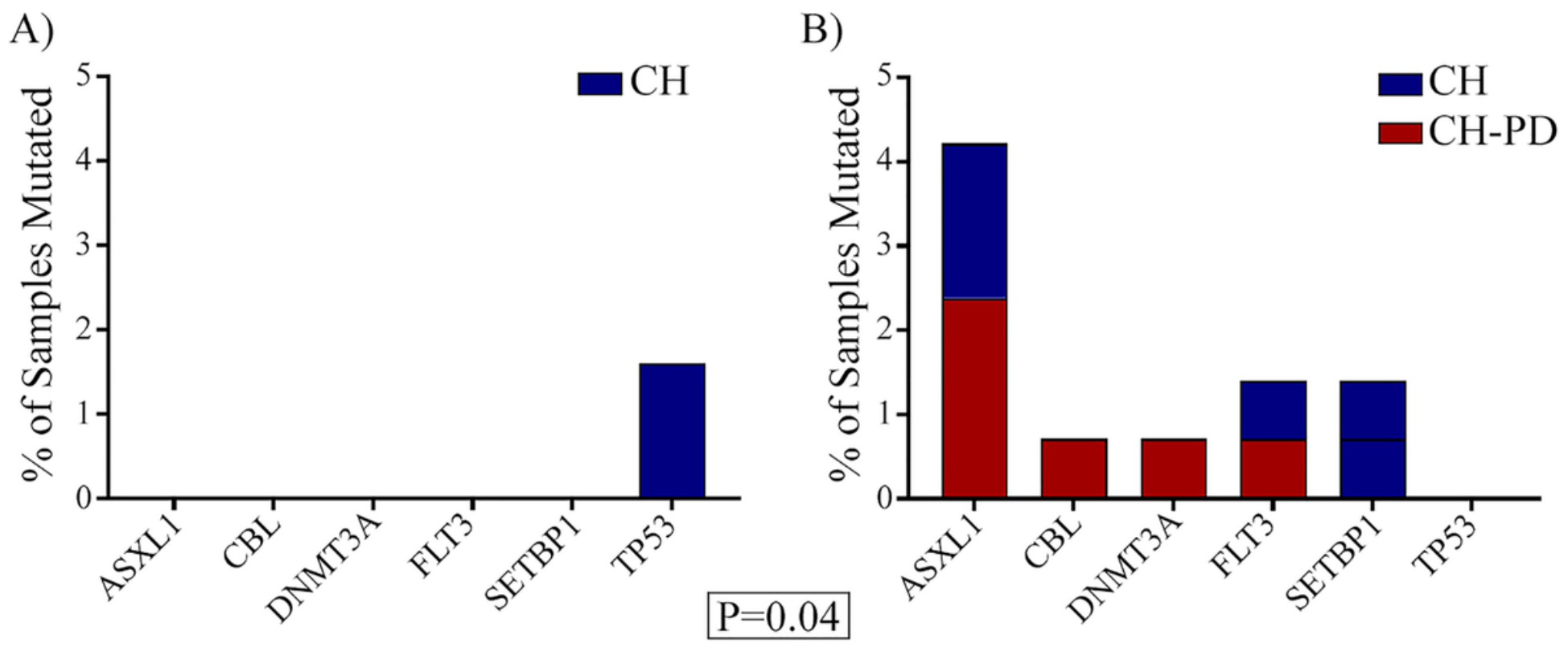


Comparison of overall mutational frequency by genes associated CHIP for each patient. Mutations are defined as "clonal hematopoiesis" $(\mathrm{CH})$, or "clonal hematopoiesis- and known to be pathogenic in a hematologic malignancy" (CH-PD); (A \& B) A, normal endothelial function group; $B$, endothelial dysfunction group.
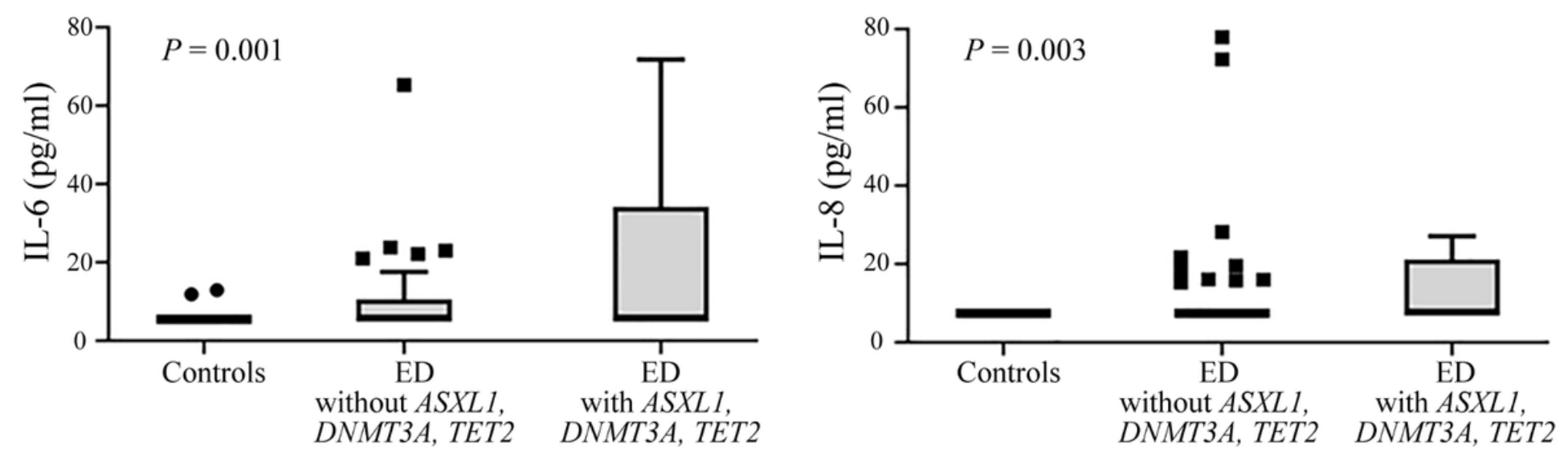

Figure 2

Plasma levels of IL- 6 and IL-8 among controls and patients with endothelial dysfunction with or without mutations in ASXL1, DNMT3A and TET2 genes. IL- 6 and IL-8 levels were elevated in patients with endothelial dysfunction and mutations in ASXL1, DNMT3A and TET2 genes $(P=0.001$ and $P=0.003$, respectively). ED, individuals with endothelial dysfunction.

\section{Supplementary Files}

This is a list of supplementary files associated with this preprint. Click to download.

- Supplementarytable1.docx 